\title{
Robot-Specific Social Cues in Emotional Body Language
}

\author{
Stephanie Embgen Matthias Luber Christian Becker-Asano Marco Ragni Vanessa Evers Kai O. Arras
}

\begin{abstract}
Humans use very sophisticated ways of bodily emotion expression combining facial expressions, sound, gestures and full body posture. Like others, we want to apply these aspects of human communication to ease the interaction between robots and users. In doing so we believe there is a need to consider what abstraction of human social communicative behaviors is appropriate for robots. The study reported in this paper is a pilot study to not offer simulated emotion but to offer an abstracted robot version of emotion expressions and an evaluation to what extent users interpret these robot expressions as the intended emotional states. To this end, we present the mobile, mildly humanized robot Daryl, for which we created six motion sequences that combine human-like, animal-like, and robot-specific social cues. The results of a user study $(\mathrm{N}=29)$ show that despite the absence of facial expressions and articulated extremities, subjects' interpretation of Daryl's emotional states were congruent with the abstracted emotion display. These results demonstrate that abstract displays of emotion that combine human-like, animal-like, and robot-specific modalities could in fact be an alternative to complex facial expressions and will feed into ongoing work identifying robot-specific social cues.
\end{abstract}

\section{INTRODUCTION}

As robots move from the factory floor into our homes and workplaces, they enter environments that are designed for humans and are inherently social by nature. To operate in everyday environments requires a humanlike morphology as well as effective and intuitive ways to communicate with humans. Emotions are an important means of communication as they are able to compactly convey a great deal of information in a short time. Fong et al. [1] state in their survey that;

"A socially interactive robot must send signals to the human in order to: (1) provide feedback of its internal state; (2) allow humans to interact in a facile, transparent manner."

Many robots that operate in human inhabited environments do not possess sophisticated facial or gestural feature capabilities. This may be due to technical

S. Embgen, M. Luber, K.O. Arras are with the Social Robotics Lab, Dept. of Computer Science, University of Freiburg, Germany. \{embgens,luber,arras\}@informatik.uni-freiburg.de.

C. Becker-Asano is with the Dept. of Computer Science, University of Freiburg, Germany. basano@informatik.uni-freiburg.de.

M. Ragni is with the Center of Cognitive Science, University of Freiburg, Germany. ragni@cognition.uni-freiburg.de

V. Evers is with the Human Media Interaction, University of Twente, the Netherlands. v.evers@utwente.nl.
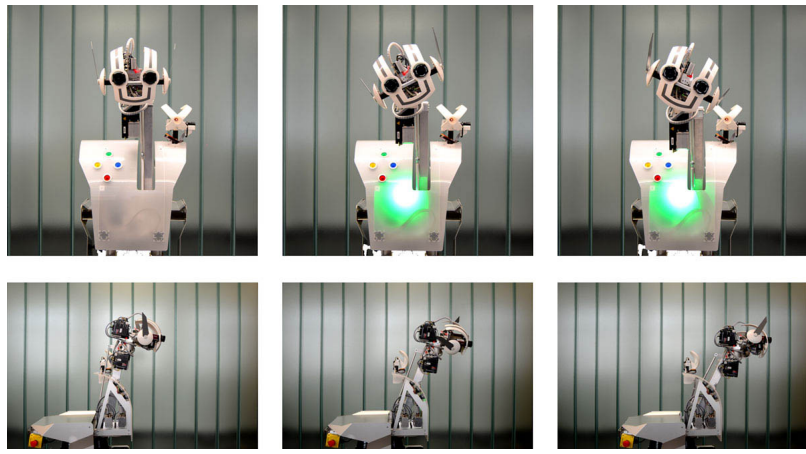

Fig. 1. Daryl performing the motion sequence curiosity.

reasons (system complexity), economic reasons (costsensitivity of marketable domestic and service robots), or application-specific requirements (robots in emergency relief situations are subject to size restraints but require easy to interpret social cues to deal with injured and traumatized people).

We argue that instead of mimicking intricate human affective communication, smooth human robot interaction requires robot-specific abstracted forms of communication that effectively communicate the congruent emotion and are experienced by the user as socially normative. Furthermore, human morphology, even if highly sophisticated, only offers a limited number of expression modalities. Since robot morphology is synthetic, new communication modalities can be explored to assess effective robot-specific ways to communicate emotion or internal states in a manner that is socially normative and natural to users. These modalities can be inspired by nature. Animals, for instance, can communicate with their ears, smells, tails and stand up the hair of their coats. Other modalities for robots are due to technology and therefore specific to robots only, such as certain sounds, light, colors, shape, or robot-specific body parts.

In this light, the issues of robot design, the conceptualization and design of new forms of expression, and their systematic evaluation are key questions towards effective and economically feasible socially enabled robots.

\section{A. Related Work}

In the attempt to give robots a humanoid shape, it appears natural to equip them with an anthropomorphic or at least zoomorphic head and face. Previous work in emotion expression for robots therefore primarily has focused on robot facial expressions [2], [3], [4], [5].

With 21 degrees of freedom the robot Kismet could 

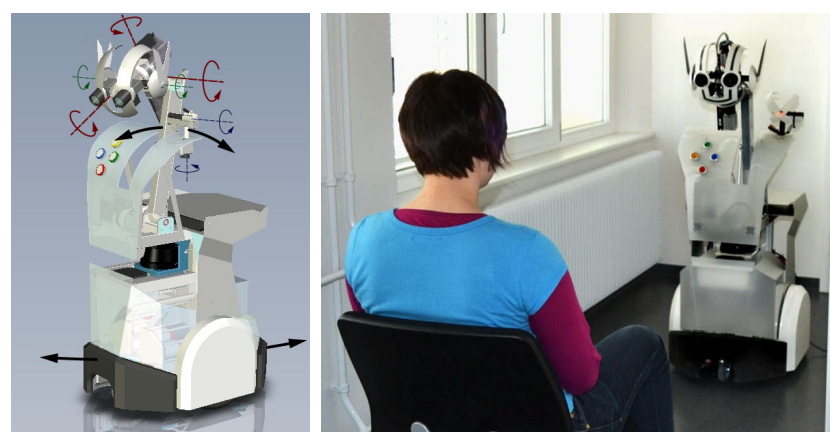

Fig. 2. Left: Robot Daryl and its ten degrees of freedom (dof): two axes to drive the robot, one to move the head forward and backward, three axes in the head for yaw, pitch, and roll, two dofs for ear-like expression modalities and two axes for a laser pointing modality with pan and tilt. Right: The experimental setup.

convey the emotions anger, disgust, fear, joy, interest, sorrow, and surprise with detection accuracies ranging from $57 \%$ up to $86 \%$, when videos of its facial movements were evaluated by seven naive observers [2]. The study also confirmed what is known from related emotion psychology, namely, that it is much more difficult to decode facial expressions of emotions from static as compared to dynamic displays.

Similarly, the Doldori mascot-type face robot [4] was designed to convey the six basic emotions anger, surprise, happiness, disgust, sadness, and fear by mapping action units from psychological literature onto seven control points of the face robot. They report recognition rates higher than $74 \%$ for the emotions sadness, happiness, anger, and surprise, but only "low recognition rates" [4] for disgust and fear.

With android robots such as Geminoid $F$ [5] the design of these machines is taken to the extreme, because they are explicitly designed to make them indistinguishable from humans at first sight [6]. An international comparison of the recognizability of the five basic emotions happy, sad, surprised, angry, and fearful (together with a neutral expression as control) modeled as static expressions for Geminoid $\mathrm{F}$ also supports the previous finding that conveying certain emotions such as fear statically is rather difficult.

Even with a simple design featuring one big eye, an eyebrow, and one rubber lip to represent its "mouth", the robot $e M u$ has been successfully employed as an "embodied emotional character" and its facial expression of happiness, sadness, and anger has been shown to have an effect on user enjoyability [3]. This gives an indication that abstracted forms of human emotion expression may very well be appropriate for robots.

Other work focusses on body movement rather than intricate facial expression [7], [8], [9], [10]. This is due to the fact that the robots that are currently on the market (such as Nao) do not have sophisticated facial expression capability. However, the need to display affect or some indication of the internal state of the robot without using

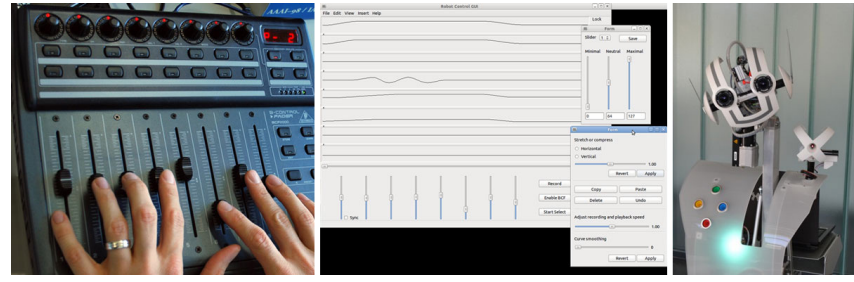

Fig. 3. The action unit editor. Motion sequences are created by playing, recording, and editing the motion of single or multiple axes using control faders of a USB/MIDI controller.

voice is clear [10], [7]. These authors pose that psychology of emotion has mainly focused on facial and vocal expressions [11], [12], although previous research has shown that emotions can be identified without speech or facial expressions [13], [14], [15]. Bernhardt and Robinson [9] showed that people associate human motion primitives with affect. Others have found that distinctive patterns of movements and postural behaviors are associated with specific emotions [16], [17].

The contribution of specific body parts or combination of body parts to the emotional expression capabilities of robots has not been systematically studied. It is thus difficult to create systems capable of generating their own emotional expressions. More precisely,there has only been very limited research [18] on the effect that moving specific joints or displaying specific motion features (from humans, animals, or robots) might have on the expressiveness of the body language being displayed.

This lack of knowledge is the main motivation for this paper where we assess the effectiveness of a unique combination of human-like, animal-like and robot-specific social cues to communicate affect. To this end, we present the robot Daryl and describe how its interaction modalities are used to display the three primary emotions happiness, sadness, and fear and the three secondary emotions curiosity, embarrassment, and disappointment.

The paper is structured as follows: the next section describes the robot and the action unit editing system to create motion sequences. Section III introduces the emotions. Section IV presents the experiment as well as the results followed by the conclusions in section V.

\section{Robot Daryl and Action Unit Editor}

Daryl is an expressive, mildly humanized mobile robot built after the belief that sustainable human robot interaction requires robot-specific abstracted forms of communication (Fig. 2). The robot has ten degrees of freedom in total and a color modality: two axes in a differential drive configuration to drive the robot, one axis in the torso that moves the entire head forward and backward, and yaw, pitch, and roll in the head. There are two earlike expression modalities on each side of the head that can turn by 360 degrees and a pan-tilt laser pointing modality on the robot's left shoulder. The translucent chest plate can be lit by an RGB-LED in any color. In 

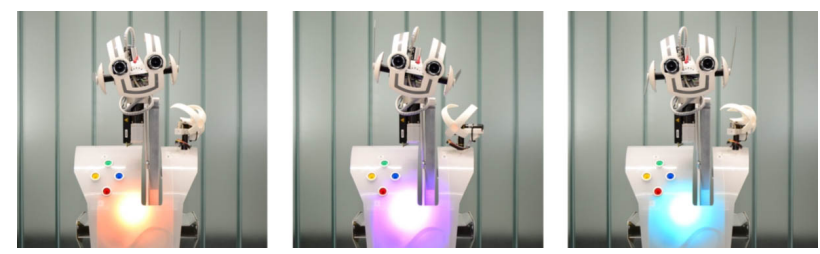

Fig. 4. Daryl performing the motion sequence happiness.

addition, the robot has four colored buttons on the chest plate and a speaker system, not used in this work.

All axis and the LED are controlled over a CAN-bus by an embedded CPU running the real-time operating system XO/2. Motion commands are sent over an API by the action unit editor running on a PC under Linux. The editing system is shown in Fig. 3. It interfaces a Behringer USB/MIDI controller through which action units composed of motion and color cues can be played and recorded on-the-fly, either for single or multiple degrees of freedom at a time, with the option to manage and manually post-process the profiles in all detail.

\section{Roвot Emotions}

In this section we describe the motion sequences with which the robot expresses the six emotions studied in this paper.

Emotion theorists distinguish between primary and secondary emotions. Primary emotions, also called basic emotions, are emotions that can be felt by humans as well as animals, whereas secondary emotions are thought of as particular to humans [19]. Opinions vary as to which emotions make up the set of basic emotions. Ekman [20] defined the set of basic emotions as consisting of the following emotions: anger, disgust, fear, happiness, sadness and surprise. Primary and secondary emotions differ in their recognizability: Primary emotions are more easily recognized and distinguished than secondary emotions [19].

In particular for robots with abstracted social cues as studied in this paper, the question is whether they are able to convey not only the universal basic emotions, but also the more complex secondary emotions. Is it necessary for the robot to have a very human-like appearance to express the full range of human emotions, or can a less humanoid robot such as Daryl achieve the same? Similarly, we combine human-like movement (articulated neck, full body movement) and modalities inspired by nature (ears) as well as robot-specific communication modalities such as light and color. This study is the first in a series of studies addressing abstracted robot-specific affect communication. In this first pilot, we combine modalities and offer an abstracted robot communication mode of emotions. In consecutive studies we aim to compare the effectiveness of human-like versus nature-inspired versus robot-specific modalities. We address these questions by designing motion sequences for
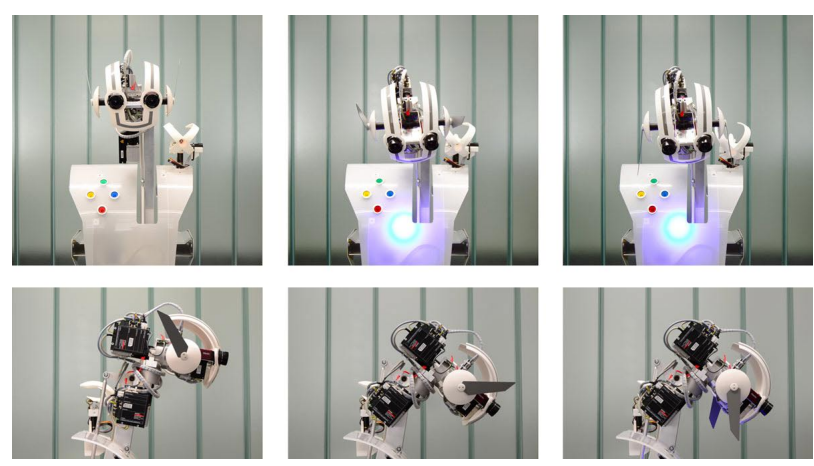

Fig. 5. Daryl performing the motion sequence sadness.

primary and secondary emotions and evaluating whether those emotions can be recognized and distinguished.

Six emotions were chosen and designed for Daryl, three of them are the basic emotions happiness, sadness and fear. To evaluate how well secondary emotions are recognized, we selected disappointment, embarrassment and curiosity. Not all psychologists classify curiosity as an emotion. It was, however, included for its potential as an interaction initiator in the same way Vikia used a display of interest to initiate interaction [21].

The emotion disappointment is very similar to the emotion sadness. Plutchik [22] describes disappointment as the emotion that combines sadness and surprise. Thus, Daryl's motion sequences for sadness and disappointment bear a certain resemblance. Both emotions were chosen despite their similarity to test how well even similar emotions can be distinguished.

Sources used for human emotional expressions were Bodily Expressions of Emotions by H.G. Wallbott [23] and the Face and Body Gesture Database (FABO) [24]. The ear movement was modeled after the way animals use their ears to express emotions. Those expressions were taken from The Expression of the Emotions in Man and Animal by Charles Darwin [25]. The movement of the pointing modality and the whole-body motion through the drive axes was taken from either human or animal emotional expression. As expression of emotions through color is unusual for both humans and animals, idiomatic references to colors like "feeling blue" or "turning red" were employed for the use of color in Daryl's emotions.

The motion sequences were designed to take place within the social space of the respective communication partner according to the Proxemics theory. Social space refers to a distance between $120 \mathrm{~cm}$ and approximately $4 \mathrm{~m}$ between two people and is the usual distance for interaction between acquaintances [26]. In the following, each emotion is illustrated by a sequence of front views of the robot, those with important lateral movements are shown with additional side views ${ }^{1}$.

\footnotetext{
${ }^{1}$ The sequences are best seen in the video that accompanies the paper. A high quality version is also available at http://www . youtube.com/user/socialrobotics
} 


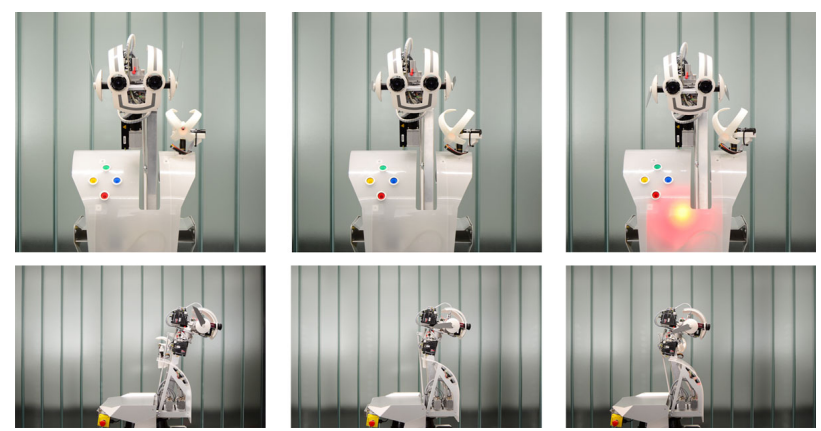

Fig. 6. Daryl performing the motion sequence fear.

Happiness: Both humans and animals show happiness through an upright posture. Wallbott describes "dancing for joy" as well as "various purposeless movements" and "head nods to and fro". Raised ears in animals are also a sign for happiness.

At the beginning of the happiness motion sequence, Daryl raises its head and ears slightly. The head starts moving from side to side. The ears alternately move back and forth to represent a dancing motion. The pointing modality moves from side to side as well in a dancing manner. The LED cycles through the colors red, purple, blue, green, yellow, orange, ending on green (see Fig. 4).

Sadness: According to Wallbott, sadness is displayed by few and slow movements and a drooping head. FABO mentions sagging shoulders and posture.

Therefore, in the motion sequence for sadness, Daryl lowers his head. The ears mirror the motion by moving forwards until they are parallel to the ground, where they pause for a moment before dropping into a position perpendicular to the ground. The dropping motion represents the overall powerlessness of the sequence. The pointing modality imitates the movement of head and ears, lowering itself before dropping into its lowest position. Throughout the whole motion sequence, the LED glows blue, a reference to the expression "feeling blue" for sadness (see Fig. 5).

Fear: A common sign for fear in animals is the flattening of the ears. Dogs in particular, as well as humans, duck their heads when scared. FABO mentions retreating as a sign of fear.

In the motion sequence for fear, Daryl quickly drives backwards and draws back its head. The ears lie flat to the side of the head. The pointing modality turns towards the neck. After retreating, the LED flashes red several times like a heart pounding with fear (see Fig. 6).

Curiosity: In the motion sequence curiosity, Daryl first drives forwards for about $15 \mathrm{~cm}$, stretching its head towards the person at the same time and tilting it to one side. One ear moves slightly forwards, the other one slightly backwards. The LED blinks once in green. Next, the robot drives forwards again, stretching its head even further and tilting it to the other side. The ears change their position as well. The LED blinks green twice. Each time the robot moves, the pointing modality makes a

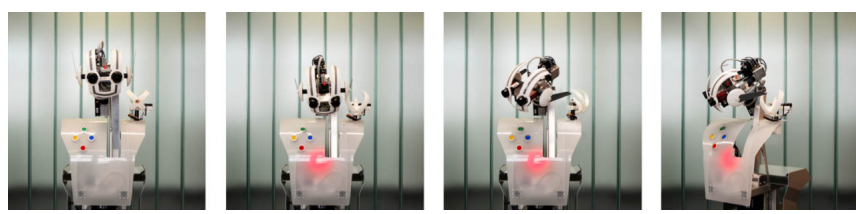

Fig. 7. Daryl performing the motion sequence embarrassment.

nodding motion (see Fig. 1). In the experiments, the distance between robot and subject was $140 \mathrm{~cm}$. As in this motion sequence, the robot drives forwards a total of $30 \mathrm{~cm}$ and stretches its head even further towards the subject, resulting in a reduction of distance of about 50 $\mathrm{cm}$. Curiosity is the only motion sequence where Daryl leaves the social space of the person and moves into its personal space.

Embarrassment: Wallbott describes the expression of the emotion embarrassment as a movement where the whole body, particularly the face, is turned away and the head lowered.

Daryl first lowers its head, drawing it back a little, with the ears lying flat to the side of the head. The pointing modality is lowered as well. Then Daryl's head and the pointing modality turn away from each other. To emphasize the "turning away" motion, the whole robot turns in the same direction as the head by about 45 degrees. The LED slowly pulsates in a dull red, imitating a human blush (see Fig. 7).

Disappointment: In the motion sequence for disappointment, Daryl shakes its head while lowering it very slowly. The ears move forwards until they are parallel to the floor. The LED glows blue throughout the whole motion sequence. The pointing modality imitates the movement of the head (see Fig. 8).

The most striking difference between the motion sequences for sadness and disappointment is the element of Daryl shaking its head in the disappointment movement. This is meant to convey that whatever elicited Daryl's sadness happened very recently.

\section{EXPERIMENTS}

The central question is, if Daryl's displayed emotions can be successfully recognized and interpreted by humans above chance. Consequently, we decided to conduct an empirical investigation. Twenty-nine students and postgraduate students (23 male/6 female) of the University of Freiburg participated in this experiment. The average age was 25.7 years with a standard deviation of 3.8 years.

\section{A. Materials, Procedure and Design}

Each participant was asked to sit on a chair facing Daryl at a distance of $1.4 \mathrm{~m}$ (see Fig. 2). As mentioned above, this distance was chosen because it lies within the social space of the subject, a common distance for people to interact who are not relatives or close friends. At a closer distance the subject might have felt threatened by the robot whereas a too large distance 


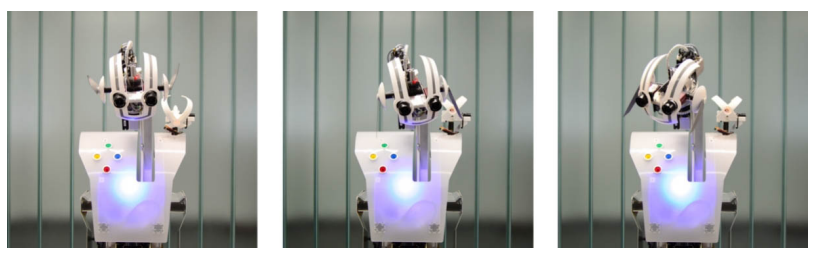

Fig. 8. Daryl performing the motion sequence disappointment.

might have made the subject feel more like an observer than a communication or interaction partner. In a next step, each participant was informed by clear instructions about the experiment - namely that Daryl will show some motion sequences - and their task - namely to assess these emotions in a questionnaire in German. The motion sequences associated with a specific emotion were presented in a randomized order. After the presentation of the motion sequence, Daryl would stay in the final position to give the participant enough time to assess this emotion in the questionnaire. The participants were asked to answer each question quickly to get an immediate and authentic evaluation of the emotions.

For each movement sequence observed, subjects had to rate the extent to which this represented nine emotions on a 5 point Likert type scale. These nine emotions included the 3 primary and 3 secondary emotions. We added three more emotions to the assessment list to avoid a biased data collection instrument. For this purpose, the three other basic emotions anger, disgust and surprise were added to the list of emotions resulting in nine options. The participants were asked to evaluate on the five point scale, how intensely they thought the emotion was expressed in the motion sequence. The order in which the emotion list was sorted was assigned according to the order in which each emotion appears on the Geneva Emotion Wheel [27], clockwise starting from the top. The five point scale was labeled according to a German translation of the Positive and Negative Affect Schedule [28] with the designators "very slightly or not at all" (later encoded by 0 ), "a little" (1), "moderately" (2), "quite a bit" (3), and "extremely" (4).

After the assessment Daryl would go back to its neutral position before the next motion sequence would start. The experiment lasted approximately 15 to 20 minutes.

\section{B. Results}

When comparing the mean over all subjects for every motion sequence, the corresponding emotion received always the highest rating. This is especially strong for the motion sequences curiosity, happiness, and fear (see Fig. 9 and Tab. I).

The motion sequence embarrassment, however, received a more ambiguous rating. While the subjects evaluated embarrassment as the most prominent emotion, the ratings for sadness, fear, and disappointment also scored high. Interestingly, the subjects were able to distinguish between the motion sequences for sadness and disappointment, despite their similarity. Still, both received high ratings for the other respectively, with all other emotions rated similarly.

In a further statistical analysis we were not only interested in if there is a significant difference between Daryl's presented emotion and all others, but if there is a clear dissociation between Daryl's emotion and the second highest rated emotion - as in nearly all cases the other emotions played no role. In other words, if we can dissociate the predicted emotion from the second strongest emotion in Tab. I, then this implies by extension that the depicted emotion is much better recognized than the rest. And, this demonstrates that the highest rated emotion is the one associated with the motion sequence. All highest and second highest rated emotions and the respective statistical test (dependent t-test for paired samples, Bonferroni corrected) can be found in Table II.

Of all motion sequences, the curiosity condition was mostly interpreted as curiosity (mean: 3.69, SD: 0.5). It was also associated with happiness (mean: 1.63, SD: 1.09). This could support the idea that the robot's curiosity was perceived as conveying friendly interest. All negative emotions, particularly anger, received very low ratings for the motion sequence curiosity. Although Daryl intrudes into the personal space of the subjects during this motion sequence, this seems to have been perceived as neither aggressive nor malicious.

The results for happiness and fear, which are both basic emotions, were considerably more clear cut than the results for embarrassment, which is not a primary emotion. Still, even embarrassment was clearly identified by the test subjects, although the emotions sadness, fear, and disappointment also scored relatively high.

The motion sequences sadness and disappointment show considerable similarities. Sadness received high ratings for disappointment and vice versa. But even though the emotions sadness and disappointment are closely related and the corresponding motion sequences

\begin{tabular}{|c|c|c|c|c|c|c|}
\hline Emotions & Hap & Sad & Fear & Cur & Emb & Dap \\
\hline Hap & $\mathbf{2 . 8 8}$ & 0.00 & 0.03 & 1.63 & 0.03 & 0.03 \\
\hline Sad & 0.00 & $\mathbf{3 . 2 9}$ & 0.31 & 0.02 & 1.48 & 1.81 \\
\hline Fear & 0.14 & 0.59 & $\mathbf{2 . 9 8}$ & 0.00 & 1.50 & 0.40 \\
\hline Cur & 1.17 & 0.03 & 0.16 & $\mathbf{3 . 6 9}$ & 0.02 & 0.14 \\
\hline Emb & 0.38 & 1.29 & 0.86 & 0.21 & $\mathbf{2 . 4 1}$ & 0.93 \\
\hline Dap & 0.03 & 2.69 & 0.40 & 0.07 & 1.29 & $\mathbf{2 . 9 1}$ \\
\hline Ang & 0.17 & 0.21 & 0.60 & 0.12 & 0.66 & 0.81 \\
\hline Dgt & 0.10 & 0.19 & 0.50 & 0.14 & 0.84 & 0.93 \\
\hline Sur & 1.14 & 0.17 & 1.00 & 0.90 & 0.17 & 0.26 \\
\hline
\end{tabular}

TABLE I

CONFUSion matrix of the MEANS OF the VAlues ASSigned to EACH MOTION SEQUENCE (COLUMNS) FOR EACH EMOTION (ROWS) By the participants. Highest values in BOLD face. 


\begin{tabular}{|c|c|c|c|c|c|c|c|}
\hline Motion sequence & Highest & Mean & 2nd-highest & Mean & Difference & Standard error & p \\
\hline Happiness & Hap & 2.879 & Cur & 1.172 & 1.707 & 0.214 & 0.000 \\
\hline Sadness & Sad & 3.293 & Dap & 2.689 & 0.603 & 0.181 & 0.001 \\
\hline Fear & Fear & 2.982 & Sur & 1.000 & 1.983 & 0.182 & 0.000 \\
\hline Curiosity & Cur & 3.689 & Hap & 1.637 & 2.052 & 0.154 & 0.000 \\
\hline Embarrassment & Emb & 2.413 & Fear & 1.500 & 0.914 & 0.292 & 0.003 \\
\hline Disappointment & Dap & 2.913 & Sad & 1.810 & 1.103 & 0.208 & 0.000 \\
\hline
\end{tabular}

TABLE II

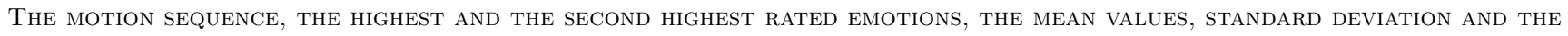
Statistical Results (Dependent t-test for paired samples, dof $=57$, Bonferroni Corrected).
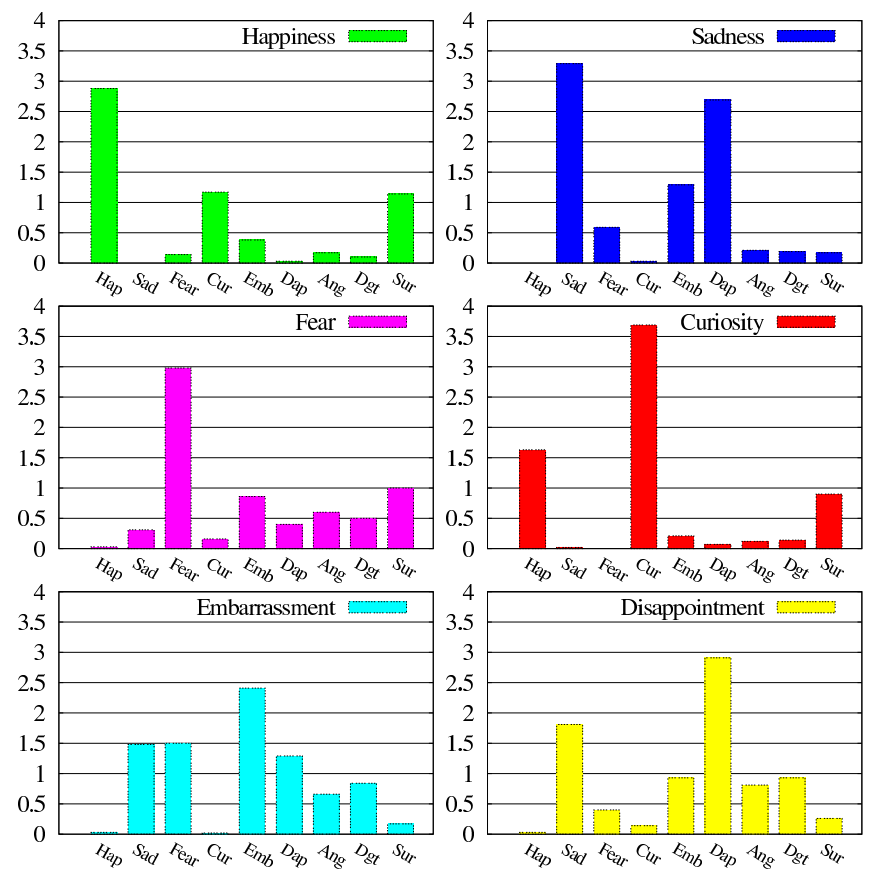

Fig. 9. Average ratings for all motion sequences. They show that the test subjects were able to correctly interpret all six emotions displayed by the robot, even those that are similar such as sadness and disappointment.

resembled each other, the test subjects were able to identify them quite reliably.

\section{Discussion}

We set out to investigate new forms of expressions to let our mildly humanized robot Daryl convey emotions. In addition, we did not limit ourselves to the standard set of primary emotions, but also included the three secondary emotions disappointment, embarrasment, and curiosity. In result, although embarrassment could be identified, the result was less clear than that achived for all other emotions. This secondary emotion seems to be harder to discern than any of the primary emotions. In addition, the primary emotion sadness was clearly distinguished from the secondary emotion disappointment. We conclude that despite their higher complexity all three secondary emotions can be conveyed by robots such as Daryl.

Furthermore, the three emotion labels which were included as distractors (bottom three rows in Table I) consistenly received very low scores. This might indicate that Daryl's motion sequences are not easily confused with other emotions.

\section{Conclusions}

We evaluated the combination of human-like, animallike, and robot-specific social cues to let Daryl express emotions in an abstract fashion. Therefore, six motion sequences were created to display three primary and three secondary emotions. A user study was conducted to analyze to what extent these robot expressions can be identified in a face-to-face setup.

The results show that the participants were able to identify the emotions displayed by the robot and were even able to distinguish between the rather similar emotions sadness and disappointment. The results clearly show the preference by participants for the correct interpretation of Daryl's emotional states, despite its inability to perform facial expressions.

These findings confirm our belief that abstract robotspecific social cues are as suited for affect communication as mimicry-based approaches of purely human or animal ways of expressing emotions. They show in particular that Daryl is an adequate platform for our ongoing research in social robotics - a field in which affective competency is clearly important.

Future work with Daryl includes, first, a more systematic comparison of the impact of human-like, animallike, and robot-specific ways of expression and, second, assessing the influence of situational context on the identifiability of Daryl's emotional expressions.

\section{ACKNOWLEDGMENT}

This work has partly been supported by the German Research Foundation (DFG) under contract number SFB/TR-8.

\section{REFERENCES}

[1] T. Fong, I. Nourbakhsh, and K. Dautenhahn, "A survey of socially interactive robots," Robotics and Autonomous Systems, vol. 24, pp. 143-166, 2004. 
[2] C. Breazeal, "Emotion and sociable humanoid robots," Int. Journal of Human-Computer Studies, vol. 59, 2003.

[3] C. Bartneck, "Interacting with an embodied emotional character," in Proc. of the Int. Conf. on Designing pleasurable products and interfaces, New York, USA, 2003, pp. 55-60.

[4] H. S. Lee, J. W. Park, and M. J. Chung, "A linear affect expression space model and control points for mascot-type facial robots," IEEE Transactions on Robotics, vol. 23, no. 5, pp. 863-873, 2007.

[5] C. Becker-Asano and H. Ishiguro, "Evaluating facial displays of emotion for the android robot Geminoid F," in IEEE SSCI Workshop on Affective Computational Intelligence, 2011, pp. $22-29$.

[6] S. Nishio, H. Ishiguro, and N. Hagita, "Geminoid: Teleoperated android of an existing person," in Humanoid Robots, New Developments. Vienna, Austria: I-Tech, 2007, pp. 343-352.

[7] M. Saerbeck and C. Bartneck, "Attribution of affect to robot motion," in Int. Conf. on Human-Robot Interaction, 2010.

[8] A. Beck, L. Cañamero, and A. Bard, "Towards an affect space for robots to display emotional body language," in Int. Sym. on Robot and Human Interactive Communication, 2010.

[9] D. Bernhardt and P. Robinson, "Detecting affect from nonstylised body motions," in Int. Conf. on Affective Computing and Intelligent Interaction, 2007.

[10] A. Beck, A. Hiolle, A. Mazel, and L. Cañamero, "Interpretation of emotional body language displayed by robots," in Affective Interaction in Natural Environments, 2010.

[11] A. Kapoor, W. Burleson, and R. Picard, "Automatic prediction of frustration," Int. Journal of Human-Computer Studies, 2007.

[12] A. Camurri, I. Lagerlöf, and G. Volpe, "Recognizing emotion from dance movement: comparison of spectator recognition and automated techniques." Int. Journal of Human-Computer Studies, vol. 59 (1-2), pp. 213-225, 2003.

[13] F. Pollick, H. Paterson, A. Bruderlin, and A. Sanford, "Perceiving affect from arm movement." Cognition, vol. 82, 2001.

[14] A. Kapur, A. Kapur, N. Virji-Babul, G. Tzanetakis, and P. Driessen, "Gesture-based affective computing on motion capture data," in Affective Computing and Intelligent Interaction, 2005.

[15] J. Lasseter, "Principles of traditional animation applied to 3d computer animation," in 14th Conf. on Computer Graphics and Interactive Techniques, 1987.

[16] K. Amaya, A. Bruderlin, and T. Calvert, "Emotion from motion," in Graphics Interface '96, Canadian Human-Computer Communications Society, 1996.

[17] L. Zhao, N. Badler, and M. Costa, "Interpreting movement manner," in Proc. of the Computer Animation, 2000.

[18] A. Brooks and R. Arkin, "Behavioral overlays for nonverbal communication expression on a humanoid robot," $A u$ tonomous Robots, vol. 22, pp. 55-74, 2007.

[19] S. Demoulin, J. P. Leyens, M. P. Paladino, R. RodriguezTorres, A. Rodriguez-Perez, and J. F. Dovidio, "Dimensions of "uniquely" and "non-uniquely" human emotions," Cognition ES Emotion, vol. 18, no. 1, pp. 71-96, 2004.

[20] P. Ekman, Handbook of Cognition and Emotion. John Wiley \& Sons, Ltd, 2005.

[21] A. Bruce, I. Nourbakhsh, and R. Simmons, "The role of expressiveness and attention in human-robot interaction," in Int. Conf. on Robotics and Automation (ICRA), vol. 4, 2002.

[22] R. Plutchik, Emotion. A psychoevolutionay synthesis. New York: Harper \& Row, 1980.

[23] H. Wallbott, "Bodily expression of emotion," European Jour nal of Social Psychology, vol. 28, no. 6, pp. 879-896, 1998.

[24] H. Gunes and M. Piccardi, "Bi-modal emotion recognition from expressive face and body gestures," Journal of Network and Computer Applications, vol. 30, no. 4, 2007.

[25] C. Darwin, The Expression of the Emotions in Man and Animals, P. Ekman, Ed. Harper Perennial, 1872/2009.

[26] E. T. Hall, The Hidden Dimension. Anchor Books, 1966.

[27] K. R. Scherer, "What are emotions? And how can they be measured?" Social Science Information, vol. 44, no. 4, 2005.

[28] H. W. Krohne, B. Egloff, C. W. Kohlmann, and A. Tausch, "Untersuchungen mit einer deutschen version der "positive and negative affect schedule" (panas)," Diagnostica, vol. 42 , no. 2 pp. 139-156, 1996. 